\title{
Risk factors for perforated marginal ulcers following pancreaticoduodenectomy and prospective analysis of marginal ulcer development
}

\author{
Andreas Minh Luu ${ }^{1 \#}$, Sina Rabea Vogel ${ }^{1 \#}$, Chris Braumann $^{1}$, Michael Praktiknjo $^{2}$, Philipp Höhn ${ }^{1 \wedge}$, \\ Sarah Förster ${ }^{3}$, Monika Janot ${ }^{1}$, Waldemar Uhl ${ }^{1}$, Orlin Belyaev ${ }^{1}$ \\ ${ }^{1}$ Department of General and Visceral Surgery, St. Josef Hospital, Ruhr-University Bochum, Gudrunstrasse 56, 44791 Bochum, Germany; \\ ${ }^{2}$ Department of Internal Medicine, University of Bonn, Venusberg-Campus 1, 53127 Bonn, Germany; ${ }^{3}$ Department of Pathology, University- \\ Hospital Bergmannsheil, Bürkle de la Camp Platz 1, 44789 Bochum, Germany \\ Contributions: (I) Conception and design: AM Luu, SR Vogel, O Belyaev, C Braumann, W Uhl; (II) Administrative support: AM Luu, SR Vogel, O \\ Belyaev, W Uhl, C Braumann, P Höhn, S Förster; (III) Provision of study materials or patients: AM Luu, SR Vogel, O Belyaev, M Praktiknjo, P \\ Höhn, S Förster; (IV) Collection and assembly of data: AM Luu, Sr Vogel, M Praktiknjo, P Höhn, S Förster; (V) Data analysis and interpretation: \\ AM Luu, SR Vogel, O Belyaev, M Praktiknjo, W Uhl, C Braumann, P Höhn, S Förster; (VI) Manuscript writing: All authors; (VII) Final approval of \\ manuscript: All authors. \\ \#These authors contributed equally to this work as first authors. \\ Correspondence to: Andreas Minh Luu, MD. St. Josef-Hospital, Ruhr-University Bochum, Gudrunstrasse 56, 44791 Bochum, Germany. \\ Email: a.luu@klinikum-bochum.de.
}

Backgroundk Perforated marginal ulcers (PMUs) are a feared long-term complication following pancreaticoduodenectomy (PD), which always require relaparotomy compared to marginal ulcers.

Methods: First, we performed a retrospective chart review for all patients who underwent PD from 2007-2016 to identify incidence and risk factors associated with PMUs. Second, we analyzed follow up gastroscopies in all patients undergoing PD from 2007-2011 to identify the overall incidence of marginal ulcers.

Results: A total of 725 patients underwent PD in the retrospective study period. 17 patients $(2.3 \%)$ suffered from PMU at a median postoperative time of 13 months. These patients were significantly younger (median age: 49 vs. 62 years; $\mathrm{P}=0.02)$ and suffered most often from chronic pancreatitis $(\mathrm{P}<0.001)$. Smoking and alcohol consumption were significantly more common $(\mathrm{P}=0.01$ and $\mathrm{P}=0.023)$. An elevated level of carcinoembryonic antigen and chronic pancreatitis were identified as independent risk factors. Overall, 373 patients were enrolled for prospective analysis. Marginal ulcers occurred in $5-5.9 \%$ over a postoperative period of 5 years.

Conclusions: Continuous treatment with proton-pump inhibitors for at least 5 years, immediate smoking cessation and follow-up gastroscopies are obligate for patients undergoing PD to avoid marginal ulcers and PMUs.

Keywords: Pancreaticoduodenectomy (PD); perforated marginal ulcer (PMU); marginal ulcer; pylorus preserving Whipple's procedure; pancreas

Submitted Oct 16, 2020. Accepted for publication Dec 13, 2020.

doi: $10.21037 /$ gs-20-763

View this article at: http://dx.doi.org/10.21037/gs-20-763

^ORCID: Andreas Minh Luu, 0000-0001-8981-8973; Philipp Höhn, 0000-0003-2913-9690. 


\section{Introduction}

Major developments in the technique of pancreaticoduodenectomy (PD) have been achieved within the last 100 years (1-4). Mortality rates of initially over $50 \%$ decreasing to less than $3 \%$ in high-volume pancreatic surgery centers nowadays reflect the formidable improvement of surgical advances $(5,6)$. Most studies in literature dealing with pancreatic surgery investigate specific complications such as postoperative pancreatic fistula (POPF), delayed gastric emptying (DGE) or postpancreatic hemorrhage (PPH) (5,7-11).

Especially regarding late-onset complications focus must be set on marginal ulcers (MU), which represent ulcers at the duodenojejunostomy or gastrojejunostomy ("GE" = gastroenterostomy for both) following pylorus-preserving PD (PPPD) or PD (Figure 1). They occur as a longterm complication and are prone to hemorrhage or even perforation if not or inadequately treated. Life-threatening perforated marginal ulcers (PMU) belong to the most feared long-term complications following PPPD/PD and must be avoided by every means. The most important prophylactic and therapeutic treatment is oral administration of proton pump inhibitors (PPI) or antihistaminics (6).

Several theories have been proposed in the pathogenesis of MU. One common theory refers to the separation of the gastric remnant or duodenum from the hepaticojejunostomy and pancreaticojejunostomy (Figure 1A). This leads to a longer distance of alkaline pancreatic secretions and alkalic bile to the GE which is consecutively more vulnerable to gastric acid (6).

$\mathrm{PMU}$ is the worst form of a MU and always requires emergency relaparotomy. Therefore, it is important to understand the risk factors associated with PMU, the correct prophylactic treatment for avoidance of PMU and the best surgical treatment.

This study analyzes incidence, management, and outcome of patients with PMU to determine individual risk factors for PMU. Furthermore, we assess the results of our prospectively performed gastroscopies of patients who underwent PPPD or PD over a postoperative period of 5 years to identify the typical postoperative period of MUdevelopment.

We present the following article in accordance with the STROBE reporting checklist (available at http://dx.doi. org/10.21037/gs-20-763).

\section{Methods}

This study contained a retrospective and a prospective analysis of clinical courses. For the retrospective analysis we performed a chart review of our prospective database for all our patients who underwent a PD or PPPD between January 2007 and December 2016 at our high-volume pancreatic surgery center (St. Josef-Hospital, RuhrUniversity Bochum). Patients with PMU were compared with a control group. Informed consent for surgery as well as for data collection and analysis was given by all patients.

Hospital records, laboratory results, operative notes, and histopathological findings were analyzed. For the prospective analysis we performed gastroscopic follow up investigations of all patients who underwent a PD or PPPD from January 2007-December 2011 over a postoperative period of 5 years to identify the incidence of gastritis, anastomositis and MU. In detail, we analyzed the gastroscopic findings of routine follow up investigations as part of our standard patient care in three postoperative periods: 1 st period: postoperative month 12,2 nd period: postoperative months $13-36,3 \mathrm{rd}$ period: postoperative months 37-60. As standard type of care we suggest a postoperative pantoprazole therapy for at least two years once a day. This study contained only data of patients being treated at our hospital. Patients being treated at another institution were prone to loss to follow-up. The study was approved by the ethics committee of the Ruhr-University Bochum, Germany (permission no. 18-6580-BR) and conducted in accordance with the Declaration of Helsinki (as revised in 2013).

\section{Perioperative and operative management}

All procedures were performed by experienced HBP surgeons. Every patient received a perioperative antibiotic prophylaxis using third generation cephalosporine and metronidazole or a second-generation quinolone in case of a penicillin allergy. Preoperatively, Octreotide was administered subcutaneously (Sandostatin $200 \mu \mathrm{g}$, Novartis, Basel, Switzerland).

\section{Technique of duodenojejunostomy and gastrojejunostomy}

The favored reconstruction type for the gastrointestinal passage in our department is an antecolic duodenojejunostomy 

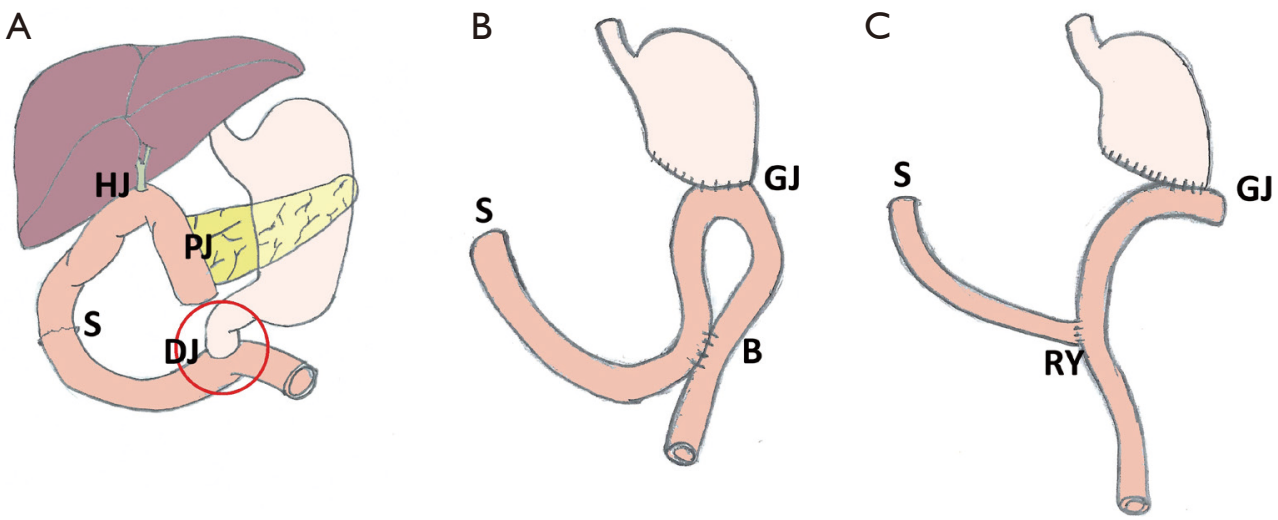

Figure 1 Schematic presentations of (A) pylorus-preserving pancreaticoduodenectomy, red circle = area of marginal ulcer development at the duodenojejunostomy (DJ), the distance between $\mathrm{HJ}$ and DJ must be at least $50 \mathrm{~cm}$; (B) standard pancreaticoduodenectomy, the distance between HJ and GJ must be at least $50 \mathrm{~cm}$; (C) new anatomic reconstruction following distal gastrectomy and resection of the gastro/duodeno-jejunostomy with construction of a new gastrojejunostomy (GJ) and Roux-en-Y anastomosis. HJ, hepaticojejunostomy; PJ, pancreaticojejunostomy; s, mesocolon slit; GJ, gastrojejunostomy; B, Braun's enterostomy.

as proposed by Traverso and Longmire (4). This anastomosis is constructed as a double layered end-toside duodenojejunostomy with two continuous sutures using an absorbable 4-0 polydioxanone suture (Figure 1A). In case of a standard PD, we applied the same technique and suture material for the gastrojejunostomy following distal gastrectomy (Figure 1B). The distance between hepaticojejunostomy and GE must be at least $50 \mathrm{~cm}$.

\section{Statistical analysis}

Percentages, median with interquartile range were used to express the data. Two-tailed chi-square tests, Fisher`s exact tests or Mann-Whitney $U$ tests were performed to compare patients with PMU to the control group. A binary logistic regression model was used for multivariate analysis to determine risk factors of PMU. All variables being significant in univariate analysis were included in the multivariate analysis. Statistical significance was present in cases of a $\mathrm{P}$ value $<0.05$. SPSS 21.0 was used for statistical analysis (IBM corp., Armonk, NY, USA).

\section{Results}

\section{Patient characteristics}

Between January 2007 and December 2016, 725 patients underwent a PD or a PPPD at our department after extensive diagnostic workup (Tables 1-3). PMU occurred in 17 patients $(2.3 \%)$ within a median postoperative period of 13 months (IQR: 8-60, Figure 2A,B, Table 4). The most common type of primary surgical procedure was PPPD (89\%) followed by PD (11\%, Table 3). No differences in terms of PMU were observed $(\mathrm{P}=0.521)$. PMU patients were significantly younger compared to the control group with a median age of 49 years versus 62 years $(\mathrm{P}=0.02$, Table 1). Consecutively, most patients were classified ASA I or II (35\% each), whereas ASA II and III (47\% and 32\%) were the predominant classifications in the other cohort. The overall male to female ratio in the PMU group was $59 \% / 41 \%$. These patients suffered predominantly from benign diseases $(71 \%)$ compared to the control group $(37 \%$, $\mathrm{P}=0.065)$. Median $\mathrm{BMI}$ was $21 \mathrm{~kg} / \mathrm{m}^{2}$ and indifferent to the control $(\mathrm{P}=0.33)$.

Chronic pancreatitis was significantly more common in patients with PMU (71\% vs. $29 \%, \mathrm{P}<0.001)$. Two third of the study group were actively smoking $(65 \%, \mathrm{P}=0.001)$, while one third was diagnosed with a history of alcohol consumption $(35 \%, \mathrm{P}=0.023)$. However, diabetes mellitus was uncommon $(6 \%$ vs. $28 \%, \mathrm{P}=0.043)$.

In the overall cohort, cardiovascular diseases were present in every second (51\%) and exocrine pancreatic insufficiency in every third patient (33\%).

Postoperative complications such as POPF, DGE, PPH, wound healing impairment, intraabdominal abscesses were not identified as risk factors for later development of PMU (Table 3). The rate of primary revision surgery or 
Table 1 Patient characteristics

\begin{tabular}{|c|c|c|c|c|}
\hline Characteristic & All patients & PMU & Control-group & $P$ value \\
\hline Sex & & & & 0.501 \\
\hline Men & $368(51 \%)$ & $10(59 \%)$ & $358(51 \%)$ & \\
\hline Women & $357(49 \%)$ & $7(41 \%)$ & $350(49 \%)$ & \\
\hline Diagnoses & & & & 0.065 \\
\hline Benign & $271(37 \%)$ & $12(71 \%)$ & $259(37 \%)$ & \\
\hline Malignant & $454(63 \%)$ & $5(29 \%)$ & 449 (63\%) & \\
\hline BMI & 24 [21-26] & $21[18-28]$ & 24 [21-26] & 0.330 \\
\hline I & $124(17 \%)$ & $6(35 \%)$ & $118(17 \%)$ & \\
\hline II & $340(47 \%)$ & $6(35 \%)$ & $334(47 \%)$ & \\
\hline III & $232(32 \%)$ & $3(18 \%)$ & $229(32 \%)$ & \\
\hline IV & $29(4 \%)$ & $2(12 \%)$ & $27(4 \%)$ & \\
\hline Cardiovascular disease & $373(51 \%)$ & $5(29 \%)$ & $368(52 \%)$ & 0.064 \\
\hline Chronic pancreatitis & $220(30 \%)$ & $12(71 \%)$ & $208(29 \%)$ & $<0.001$ \\
\hline Exocrine pancreatic insufficiency & $242(33 \%)$ & $3(18 \%)$ & 239 (34\%) & 0.152 \\
\hline Diabetes mellitus & 199 (27\%) & $1(6 \%)$ & $198(28 \%)$ & 0.043 \\
\hline
\end{tabular}

Characteristics of all patients, patients with perforated marginal ulcer and control group. PMU, perforated marginal ulcer; IQR, interquartile range; BMI, body mass index; ASA, American Society of Anesthesiologists; CBD, common bile duct.

Table 2 Preoperative diagnostic studies

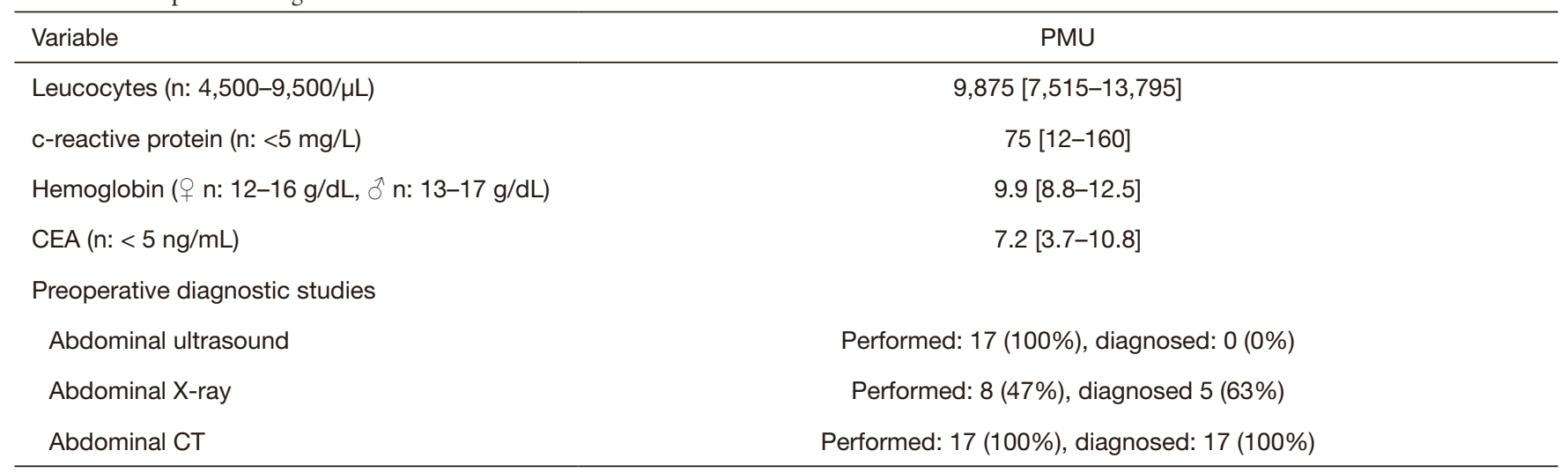

PMU, perforated marginal ulcer; n, normal; CEA, carcinoembryonic antigen; CT, computed tomography. 
Table 3 Perioperative data of primary surgery

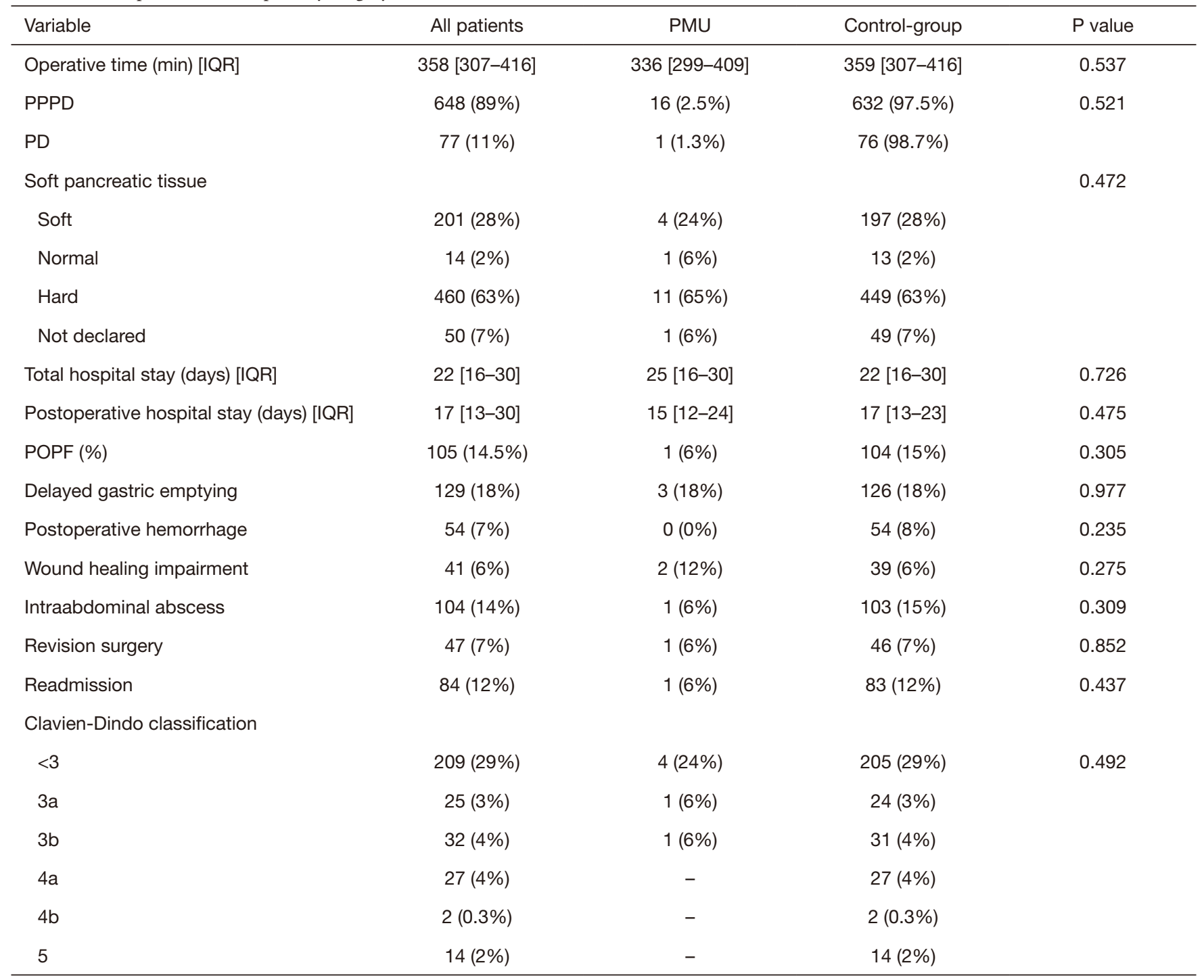

PMU, perforated marginal ulcer; IQR, interquartile range; PPPD, pylorus-preserving pancreaticoduodenectomy; PD, pancreaticoduodenectomy; POPF, postoperative pancreatic fistula.

readmission did also not contribute to PMU.

\section{Perioperative data}

Most patients with PMU had significant elevation of inflammatory values (Table 2). Median leucocyte count was $9,875 / \mu \mathrm{L}$ (IQR: $7,515-13,795 / \mu \mathrm{L}$ ), median level of c-reactive protein was $75 \mathrm{mg} / \mathrm{L}$ (normal range: $<5 \mathrm{mg} / \mathrm{L}$ ). Median level of CEA was $7.2 \mathrm{ng} / \mathrm{mL}$ (normal range: <5). A pathological CEA value was significantly more common in PMU patients $(\mathrm{P}=0.002)$. Median CEA value of the control group was $2.6 \mathrm{ng} / \mathrm{mL}(1.7-4.9 \mathrm{ng} / \mathrm{mL})$. CT-scan detected PMU in all cases $(100 \%$, Figure $3 A)$, followed by abdominal $\mathrm{X}$-ray $(63 \%$, Figure 3B). PMU was never diagnosed with abdominal ultrasound.

Intraoperative findings revealed that all perforations were located at the mesenteric side of the jejunum on the opposite side of the GE.

Most common surgical treatment in cases of PMU was resection of the GE including distal gastrectomy with construction of a gastrojejunostomy and jejuno-jejunostomy in $88 \%$ (Figure 1C; Table 4). Two patients underwent 

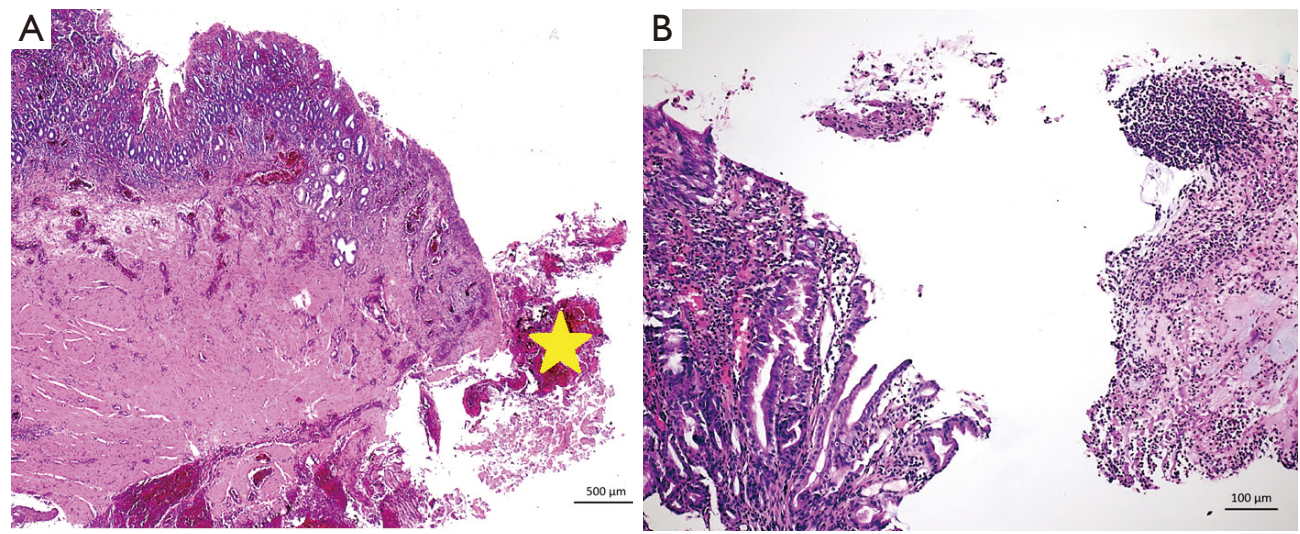

Figure 2 Histopathological findings stained with hematoxylin and eosin. (A) A patient with PMU who underwent distal gastrectomy with resection of the gastroenterostomy. A large ulceration of the gastric mucosa, transmural inflammation and perforation at the anastomosis can be seen here (yellow star). (B) Biopsy of the gastric antrum with ulceration of mucosa with regeneration of epithelium (left hand) and fibrinoid necrosis (right hand). PMU, perforated marginal ulcer.

Table 4 Specific data of patients with perforated marginal ulcer

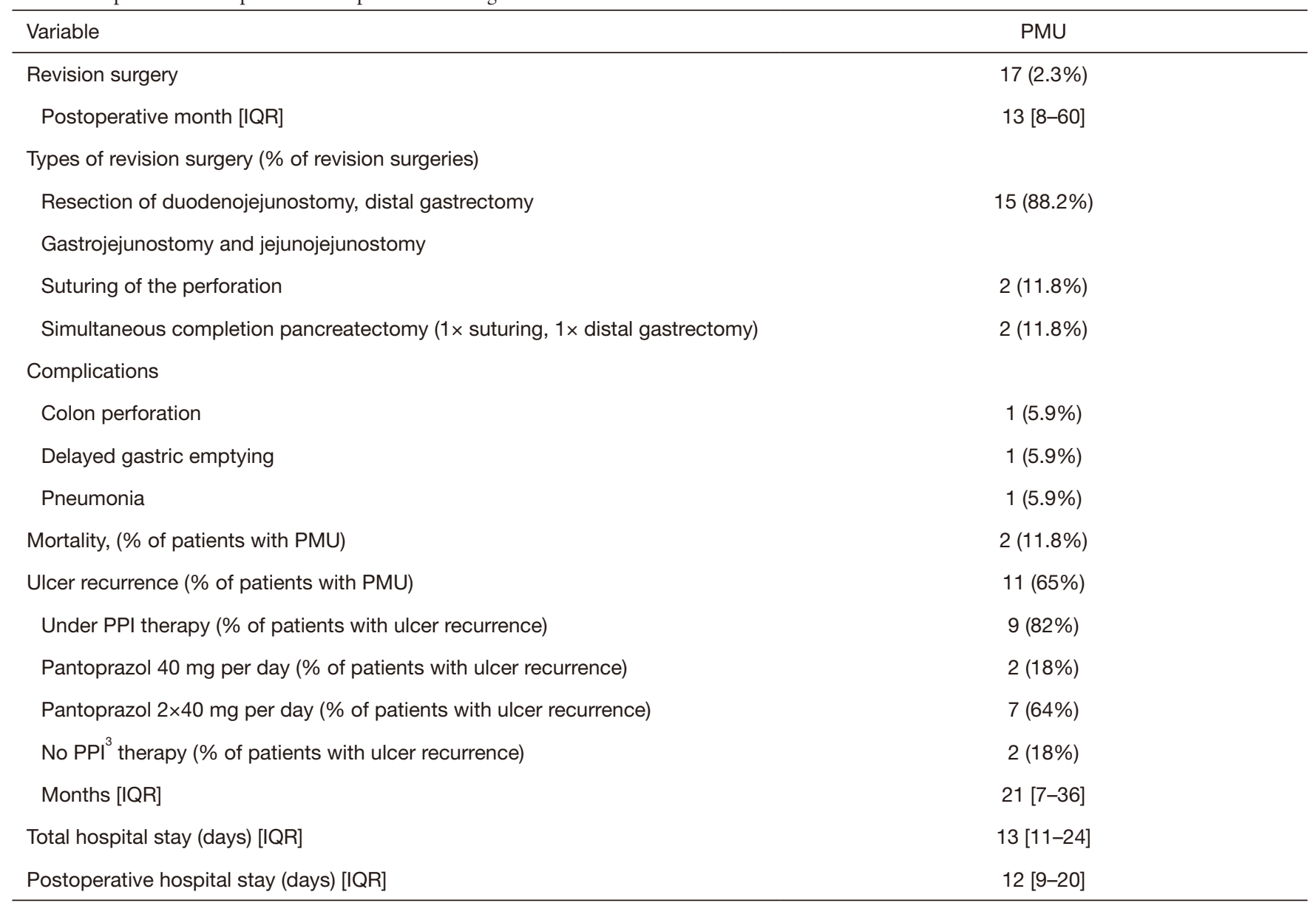

PMU, perforated marginal ulcer; IQR, interquartile range; PPI, proton pump inhibitor. 

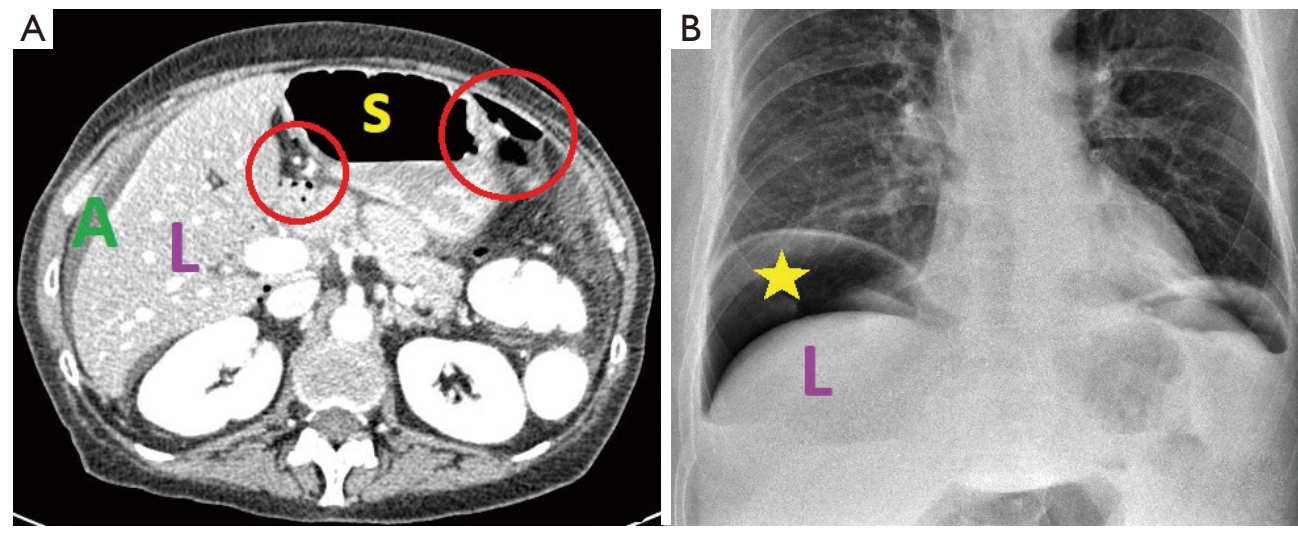

Figure 3 Radiologic findings demonstrating perigastric and subdiaphragmatic free gas representing indirect signs of perforated marginal ulcer. (A) computed tomography of a 42-year-old patient with a perforated marginal ulcer following pylorus-preserving pancreaticoduodenectomy due to chronic pancreatitis. The leaked intraabdominal air next to the duodenojejunal anastomosis and stomach (s) is encircled in red color; (B) abdominal X-ray of the previous patient demonstrating free intraabdominal air (yellow star). A detailed location of the perforation is not possible with this examination. A, ascites; L, liver.

Table 5 Multivariate analysis of variables being statistically significant in univariate analysis

\begin{tabular}{llc}
\hline $\begin{array}{l}\text { Influencing variables } \\
\text { for PMU }\end{array}$ & P value & $\begin{array}{c}\text { Odds ratio }(95 \% \\
\text { confidence interval) }\end{array}$ \\
\hline Alcohol consumption & 0.908 & \\
Smoking & 0.164 & \\
Pathological CEA value & 0.003 & $4.482(1.648-12.193)$ \\
Age & 0.773 & \\
Sex & 0.961 & \\
Chronic pancreatitis & 0.001 & $5.866(2.005-17.156)$ \\
Diabetes mellitus & 0.041 & $0.118(0.015-0.917)$ \\
\hline
\end{tabular}

$\mathrm{PMU}$, perforated marginal ulcer; CEA, carcinoembryonic antigen.

suturing of the perforation (11.8\%). Simultaneous completion pancreatectomy was required in one patient of each group. Postoperative complications occurred rarely $(6 \%)$ and were unspecific (colon perforation, DGE and pneumonia). Two patients died due to fulminant sepsis (11.8\%). Median total hospital stay was 13 days (11-24), postoperative hospital stay was 12 days (9-20).

Postoperatively, most patients were sticking to PPI therapy (82\%). However, ulcer recurrence occurred in two thirds of the patients $(65 \%)$. Most were under double dosed PPI treatment as recommended by us (64\%, Pantoprazole $40 \mathrm{mg} 2 \times /$ day). Only two patients were not compliant and did not stick to PPI treatment. Median time to postoperative development of ulcer recurrence was 21 months.

\section{Multivariate analysis}

All variables showing statistically significant differences between PMU patients and the control group (alcohol consumption, smoking, pathological CEA value, age, sex, chronic pancreatitis, and diabetes mellitus) were investigated in a multivariate binary logistic regression analysis (Table 5). We further added the variable "sex", which was not significant in univariate analysis to identify whether this variable can be identified as an independent risk factor. A pathological CEA value and chronic pancreatitis were independent risk factors. Conversely, diabetes mellitus was negatively associated with development of PMU.

\section{Gastroscopic follow-up}

Overall, 373 patients were enrolled for prospective gastroscopic follow up investigations after PPPD and PD. However, we observed a high rate of patients who did not undergo regular follow up investigations as required and suggested. Therefore, we investigated the total number of gastroscopic findings for each of the three follow-up periods as demonstrated in Table 6. The most common gastroscopic finding in all periods was an unspecific gastritis (Figure 4). Standard treatment was pantoprazole $40 \mathrm{mg}$ once a day. Marginal ulcers occurred in $5-5.9 \%$ over a period 
Table 6 Prospectively evaluated gastroscopic findings of patients following (pylorus-preserving) pancreaticoduodenectomy

\begin{tabular}{|c|c|c|c|c|c|}
\hline $\begin{array}{l}\text { Gastroscopic follow up } \\
\text { (total No. patients N=373) }\end{array}$ & Regular finding & Gastritis & Anastomositis & Marginal ulcer & $\begin{array}{c}\text { Perforated } \\
\text { marginal ulcer }\end{array}$ \\
\hline p.o. month $12(\mathrm{~N}=255)$ & $17(6.7 \%)$ & $202(79 \%)$ & $19(7.5 \%)$ & $15(5.9 \%)$ & $2(0.8 \%)$ \\
\hline p.o. months $13-36(\mathrm{~N}=233)$ & $8(3.4 \%)$ & $184(79 \%)$ & $30(12.9 \%)$ & $11(4.7 \%)$ & - \\
\hline p.o. months $37-60(\mathrm{~N}=202)$ & $9(4.5 \%)$ & $162(80.2 \%)$ & $21(10.3 \%)$ & $10(5 \%)$ & - \\
\hline
\end{tabular}

p.o., postoperative.

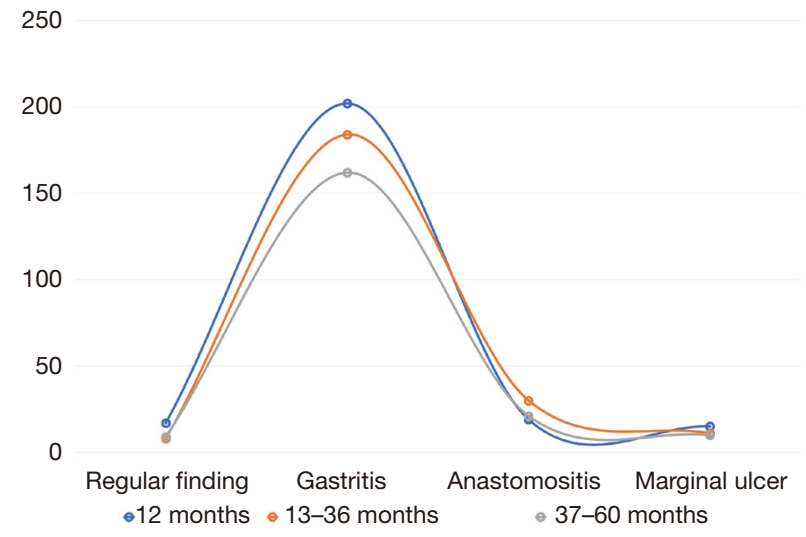

Figure 4 Graph demonstrating gastroscopic findings as part of the prospective analysis over a postoperative period of 6 years. Blue line: findings at months 12 , orange line: findings from months 13-36 and grey line: findings from months 37-60.

of 5 years (Figures 4,5A). They were always treated with pantoprazole $40 \mathrm{mg}$ twice a day for at least 6 weeks. Follow up gastroscopy after 6 weeks determined the continuation of the double dose therapy or whether reduction to pantoprazole $40 \mathrm{mg}$ once a day was feasible. No rise or decrease of MU-incidence were observed within 60 months postoperatively. Anastomositis occurs less frequently in the first year $(7.5 \%)$ compared to the following 5 years $(12.9 \%$ and $10.3 \%$, Table 6).

\section{Discussion}

Already in the early days of PD, MU have been identified as serious and potentially life-threatening long-term complications. Allen O. Whipple's 1940 published variant of a PD with antrectomy was modified with a hemigastrectomy by Charles G. Child in 1966 to reduce the rate of gastrin secretion and therefore leading to a lower gastric acid level with a consecutively lower risk for MU $(1,12)$. Nowadays, Child's modification of Whipple's procedure is generally considered as standard PD (Figure 1B). To avoid symptoms such as dumping syndrome or dyspepsia due to the loss of sphincter and reservoir, Traverso and Longmire reported their experience with the technique of PPPD in 1978 (Figure 1A) (4). Their results of a significantly lower rate of dyspepsia and no difference in MU-rates were confirmed by the studies of Klinkenbijl et al. in 1992 and recently by Park et al. in 2020 (4,6,13). Also, in cases of PMU we observed no differences between PD and PPPD. Nowadays, PPPD has become a worldwide established and most often favored technique in pancreatic surgery $(6,13,14)$.

According to current literature, the overall postoperative rate of MU ranges from $5.4 \%$ to $14.3 \%(6,15-18)$. Our prospective analysis confirms that MU occur rarely but continuously in around $5 \%$ following PPPD/PD over a period of 5 years (Figure $5 A$ ).

PMU represents the worst and most life-threatening form occurring in up to $8.3 \%$ according to literature (Figure 5B) $(6,16,18)$. Our incidence rate is significantly lower with $2.3 \%$, most likely because our patients undergo structured postoperative follow-up gastroscopies to identify and treat $M U$ in time. This demonstrates the major importance of operative, peri- and even postoperative treatment in a specialized high-volume pancreatic surgery center. Analog to literature, our data confirm that PMU most often occur after 1 year. Interestingly, it seems that the typical location for PMU is the mesenteric jejunal side on the opposite side of the GE. Maybe minor mesenteric disturbances of blood circulation associated with smoking or cardiovascular diseases facilitate ulcer development. Patients may be prone to PMU within the first year due to the new anatomic situation or increased postoperative stress levels. On the other hand, we observed a biphasic trend of MU occurrences in PMU patients. Two third of the patients with PMU suffer from MU recurrence around 21 months after emergency surgery even under PPI-treatment. Therefore, the physician must stay aware and continue follow up investigations to diagnose and treat $\mathrm{MU}$ in time. 

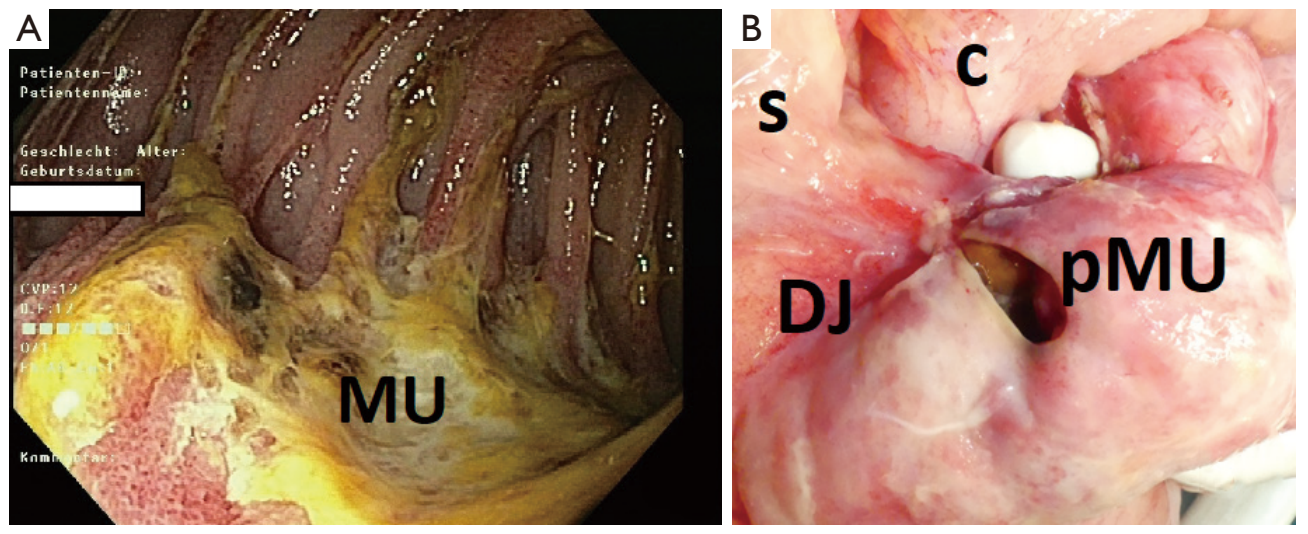

Figure 5 Gastroscopic finding of marginal ulcer and intraoperativ demonstration of perforated marginal ulcer. (A) gastroscopic demonstration of a severe marginal ulcer $(\mathrm{MU})$ of a 46-year-old patient with chronic pancreatitis 12 months following pylorus preserving pancreaticoduodenectomy. (B) Intraoperative demonstration of a 42-year-old patient with a perforated marginal ulcer (PMU) following pylorus-preserving pancreaticoduodenectomy due to chronic pancreatitis. DJ, duodenojejunostomy; s, stomach; c, transverse colon.

Renewal of the GE is the most common surgical approach followed by suturing of the perforation (Figure 1C). Most PMU are too large for suturing which explains the low incidence of performed suturing in this study. MU can be oligosymptomatic presenting with symptoms such as nausea, upper GI bleeding, anemia, or abdominal pain. Park et al. reported melena as a primary symptom in $50 \%$ which can be treated endoscopically in $21 \%$ (6). As our prospective analysis demonstrates, MUs are most commonly even asymptomatic. As most studies diagnosed MU in symptomatic patients only, asymptomatic patients were not detected. Therefore, the rates of $M U$ in our prospectively performed study most likely represent a more realistic incidence rate. In contrast to $\mathrm{MU}$, patients with PMU typically present in the emergency room with acute onset of severe abdominal pain.

Most patients with PMU are significantly younger than the control group, because most suffered from chronic pancreatitis being the more common cause for surgery in younger compared to elderly who typically undergo surgery due to malignant disease. Chronic pancreatitis, alcohol consumption and smoking are major risk factors for PMU. As smoking has been identified as an independent factor for elevation of CEA levels apart from malignant disease, our data confirm that elevated CEA levels represent a marker for chronic inflammation $(19,20)$. They have been identified as independent risk factors for PMU in our study. Patients with diabetes mellitus are less often affected, most likely because diabetes mellitus occurs more commonly in elderly patients, whereas PMU patients tend to be younger and suffer less likely from diabetes.

To understand the pathophysiological mechanisms of MU development it is important to reconsider the new constructed anatomy following PD/PPPD. As Figure 1 demonstrates, the gastroduodenal passage is replaced by a duodeno- or gastrojejunal passage. Therefore, alkalic pancreatic bicarbonate or bile drains into the jejunum at least $50 \mathrm{~cm}(19.7 \mathrm{inch})$ apart from the duodeno- or gastrojejunostomy, which causes the vulnerability of the GE to gastric acid.

Secretion of gastrin inhibiting factors like somatostatin or secretin is usually stimulated by a lower $\mathrm{pH}$-value in the duodenum but not in patients with PPPD and PD $(15,21)$. Other protective factors against ulcers are the Brunner glands in the duodenal bulb, which secrete protective mucus and bicarbonate to neutralize gastric acid (16). Long-term factors refer to postoperative stenosis of the pancreaticojejunostomy or exocrine pancreatic insufficiency, which results in a lower production or local effectiveness of alkaline pancreatic secretion at the GE (6).

Common types of the GE are the reconstruction of Traverso/Longmire and the Roux-en-Y (R-Y) reconstruction [1978] (4). Studies demonstrated a higher rate of MU in patients with $\mathrm{R}-\mathrm{Y}$ anastomosis, which is caused by direct exposition of the jejunal loop to gastric juice without neutralization from an oral jejunal limb $(16,22)$. Therefore, the standard type of reconstruction at our institution is Traverso and Longmire's technique of PPPD (Figure 1A). Important technical risk factors contributing to $\mathrm{MU}$ of gastric bypasses are reduced blood 
perfusion, tension of the anastomosis and intolerance to suture material $(17,22,23)$. Patient related risk factors are helicobacter pylori infection and intake of non-steroidal antirheumatic drugs (23).

Most studies investigating $\mathrm{MU}$ focus on incidence and risk factors of $\mathrm{MU}(6,13,16,17)$. Our study shows the importance of a thorough diagnostic workup for patients with abdominal pain. Since abdominal $x$-ray and ultrasound are unable to detect all cases of PMU, it is always crucial to perform an abdominal CT in patients with an acute abdomen and elevated inflammatory parameters after PPPD/PD (Figure 3A).

PPI are considered the first-choice treatment for peptic ulcers, helicobacter pylori infections and ZollingerEllison syndrome (24). They are available since 1989 and work by irreversible inhibition of the $\mathrm{H}^{+} / \mathrm{K}^{+}$ATPase of the parietal cells of the stomach. Routinely postoperative administration together with NSAID intends to antagonize gastrotoxic effects and to reduce the rate of stress ulcers. Furthermore, stasis of gastric acid at the GE due to DGE has been estimated to contribute to MU development (6). Several concerns regarding long-term treatment with PPI have been stated in literature. Gastric atrophy, clostridium difficile infection, pneumonia, increased risk for dementia, chronic renal failure as well as malabsorption have been discussed $(23,25-27)$. However, the quality of these studies is compromised. A recently published review by Freedberg et al. investigating long-term PPI effects reported a low to very low rate of adverse effects (26). They are usually well tolerated and have a highly effective potential to reduce ulcer related upper GI bleedings $(17,26)$. We did not observe any side effects in our cohort with long-term PPI treatment.

Park et al. recommend a postoperative PPI-treatment for at least 4 months (6). Regarding the biphasic peak of symptomatic $\mathrm{MU}$, and a wide interquartile occurrence range of PMU from 8-60 months, we suggest a continuous PPI-treatment for at least 60 months postoperatively. Pantoprazole $40 \mathrm{mg}$ once a day is sufficient in most caseseven in cases of gastritis. However, those patients require control gastroscopy after 8 weeks. If the finding worsens and the patient develops a MU, a double dosed pantoprazole therapy must be initiated. Since the prices for PPI have dropped significantly within the past decades, pantoprazole for instance has become an affordable medication. With average costs of 3.55-5.99 Euro/14 tablets in German pharmacies, long-term PPI treatment with annually costs between 92.55 Euro and 156.17 Euro is significantly cheaper compared to stationary patient care and revision surgery regardless of life-threatening PMU.

As MU tend to reoccur in 2 of 3 cases regardless of PPI treatment, it seems that single systemic PPI therapy is not enough once a patient suffered from a MU. Currently, the pathophysiological cause for therapy refractory ulcers remains unclear. Therefore, we suggest adding antacids such as aluminiumhydroxid or calcium salts to the high dose PPI-treatment in every patient with PMU (28). Future studies will have to demonstrate whether the additional topical effects of antacids neutralizing the gastric acid in the stomach and at the GE will reduce the incidence of MU and PMU. Since antihistaminics can be also be used effectively for treatment of peptic ulcers, future studies should also investigate the effects of prophylactic therapy with antihistaminics compared to PPIs.

This study is limited by the circumstance that some patients were lost to long-term follow up. Some patients died in the meantime due to malignant disease or could not be contacted. Due to patient demise or lacking documentation it was furthermore difficult to analyze whether patients with PMU were treated with PPI and/or NSAID preoperatively. As Helicobacter pylori is a well-known risk factor for MU, future studies should also investigate the incidence of H.P. infections in patients with MU.

As we know, surgical expertise plays a major role in terms of short- and long-term outcome following PPPD/ PD (5,29). This study demonstrates that patient treatment cannot be considered finished with the patient discharge. Continuous treatment with PPI, immediate smoking cessation and follow-up investigations are obligate for patients undergoing PD to avoid marginal ulcers or PMU to avoid long-term postoperative morbidity and mortality.

\section{Acknowledgments}

Funding: None.

\section{Footnote}

Reporting Checklist: The authors have completed the STROBE reporting checklist. Available at http://dx.doi. org/10.21037/gs-20-763

Data Sharing Statement: Available at http://dx.doi. org/10.21037/gs-20-763

Conflicts of Interest: All authors have completed the ICMJE 
uniform disclosure form (available at http://dx.doi. org/10.21037/gs-20-763). The authors have no conflicts of interest to declare.

Ethical Statement: The authors are accountable for all aspects of the work in ensuring that questions related to the accuracy or integrity of any part of the work are appropriately investigated and resolved. The study was conducted according in accordance with the Declaration of Helsinki (as revised in 2013). The study was approved by the ethics committee of the Ruhr-University Bochum, Germany (permission no. 18-6580-BR). Informed consent for surgery as well as for data collection and analysis was given by all patients.

Open Access Statement: This is an Open Access article distributed in accordance with the Creative Commons Attribution-NonCommercial-NoDerivs 4.0 International License (CC BY-NC-ND 4.0), which permits the noncommercial replication and distribution of the article with the strict proviso that no changes or edits are made and the original work is properly cited (including links to both the formal publication through the relevant DOI and the license). See: https://creativecommons.org/licenses/by-nc-nd/4.0/.

\section{References}

1. Are C, Dhir M, Ravipati L. History of pancreaticoduodenectomy: early misconceptions, initial milestones and the pioneers. HPB (Oxford) 2011;13:377-84.

2. Griffin JF, Poruk KE, Wolfgang CL. Pancreatic cancer surgery: past, present, and future. Chin J Cancer Res 2015;27:332-48.

3. Hunt DR, McLean R. Pylorus-preserving pancreatectomy: functional results. Br J Surg 1989;76:173-6.

4. Traverso LW, Longmire WP Jr. Preservation of the pylorus in pancreaticoduodenectomy. Surg Gynecol Obstet 1978;146:959-62.

5. Luu AM, Krasemann L, Fahlbusch T, et al. Facing the surgeon's nightmare: Incidence and management of postoperative pancreatic fistulas grade $\mathrm{C}$ after pancreaticoduodenectomy based on the updated definition of the International Study Group of Pancreatic Surgery (ISGPS). J Hepatobiliary Pancreat Sci 2020;27:171-81.

6. Park Y, Hwang DW, Lee JH, et al. Analysis of Symptomatic Marginal Ulcers in Patients Who Underwent Pancreaticoduodenectomy for Periampullary Tumors. Pancreas 2020;49:208-15.
7. Balzano G, Pecorelli N, Piemonti L, et al. Relaparotomy for a pancreatic fistula after a pancreaticoduodenectomy: a comparison of different surgical strategies. HPB (Oxford) 2014;16:40-5.

8. Hackert T, Buchler MW. Management of postoperative pancreatic fistula. Chirurg 2015;86:519-24.

9. Mañas-Gómez MJ, Rodriguez-Revuelto R, Balsells-Valls J, et al. Post-pancreaticoduodenectomy hemorrhage. Incidence, diagnosis, and treatment. World J Surg 2011;35:2543-8.

10. Luu AM, Braumann C, Uhl W, et al. Does autologous fibrin sealant (vivostat((c))) reduce the incidence of postoperative pancreatic fistula after distal pancreatectomy?-a matched pairs analysis. Acta Chir Belg 2019:1-7.

11. Luu AM, Aghalarov I, Braumann C, et al. Modified SingleLoop Reconstruction for Pancreaticoduodenectomy. J Vis Exp 2019;(151). doi: 10.3791/59319.

12. Child CG 3rd, Frey CF. Pancreaticoduodenectomy. Surgical grand rounds--University of Michigan. Surg Clin North Am 1966;46:1201-13.

13. Klinkenbijl JH, van der Schelling GP, Hop WC, et al. The advantages of pylorus-preserving pancreatoduodenectomy in malignant disease of the pancreas and periampullary region. Ann Surg 1992;216:142-5.

14. Grace PA, Pitt HA, Longmire WP. Pylorus preserving pancreatoduodenectomy: an overview. Br J Surg 1990;77:968-74.

15. Kim HC, Suzuki T, Kajiwara T, et al. Exocrine and endocrine stomach after gastrobulbar preserving pancreatoduodenectomy. Ann Surg 1987;206:717-27.

16. Sakaguchi T, Nakamura S, Suzuki S, et al. Marginal ulceration after pylorus-preserving pancreaticoduodenectomy. J Hepatobiliary Pancreat Surg 2000;7:193-7.

17. Wu JM, Tsai MK, Hu RH, et al. Reflux esophagitis and marginal ulcer after pancreaticoduodenectomy. J Gastrointest Surg 2011;15:824-8.

18. Sulieman I, Strobel O, Scharenberg C, et al. Symptomatic marginal ulcer after pancreatoduodenectomy. Surgery 2020;168:67-71.

19. Sajid KM, Parveen R, Durre S, et al. Carcinoembryonic antigen (CEA) levels in hookah smokers, cigarette smokers and non-smokers. J Pak Med Assoc 2007;57:595-9.

20. Alexander JC, Silverman NA, Chretien PB. Effect of age and cigarette smoking on carcinoembryonic antigen levels. JAMA 1976;235:1975-9.

21. Prosapio JG, Sankar P, Jialal I. Physiology, Gastrin. 
StatPearls. Treasure Island (FL) 2020.

22. Steinemann DC, Bueter M, Schiesser M, et al. Management of anastomotic ulcers after Roux-en-Y gastric bypass: results of an international survey. Obes Surg 2014;24:741-6.

23. Ying VW, Kim SH, Khan KJ, et al. Prophylactic PPI help reduce marginal ulcers after gastric bypass surgery: a systematic review and meta-analysis of cohort studies. Surg Endosc 2015;29:1018-23.

24. Shi S, Klotz U. Proton pump inhibitors: an update of their clinical use and pharmacokinetics. Eur J Clin Pharmacol 2008;64:935-51.

25. Jaynes M, Kumar AB. The risks of long-term use of proton pump inhibitors: a critical review. Ther Adv Drug Saf 2018;10:2042098618809927.

Cite this article as: Luu AM, Vogel SR, Braumann C, Praktiknjo M, Höhn P, Förster S, Janot M, Uhl W, Belyaev O. Risk factors for perforated marginal ulcers following pancreaticoduodenectomy and prospective analysis of marginal ulcer development. Gland Surg 2021;10(2):739-750. doi: 10.21037 /gs-20-763
26. Freedberg DE, Kim LS, Yang YX. The Risks and Benefits of Long-term Use of Proton Pump Inhibitors: Expert Review and Best Practice Advice From the American Gastroenterological Association. Gastroenterology 2017;152:706-15.

27. Azagury DE, Abu Dayyeh BK, Greenwalt IT, et al. Marginal ulceration after Roux-en-Y gastric bypass surgery: characteristics, risk factors, treatment, and outcomes. Endoscopy 2011;43:950-4.

28. Salisbury BH, Terrell JM. Antacids. StatPearls. Treasure Island (FL) 2020.

29. Enomoto LM, Gusani NJ, Dillon PW, et al. Impact of surgeon and hospital volume on mortality, length of stay, and cost of pancreaticoduodenectomy. J Gastrointest Surg 2014;18:690-700. 